\title{
Some Jacobi Matrices with Decaying Potential and Dense Point Spectrum
}

\author{
Barry Simon* \\ Departments of Mathematics and Physics, California Institute of Technology, \\ Pasadena, CA 91125, USA
}

\begin{abstract}
We discuss doubly infinite matrices of the form $M_{i j}=\delta_{i, j+1}+\delta_{i, j-1}$ $+V_{i} \delta_{i j}$ as operators on $\ell^{2}$. We present for each $\varepsilon>0$, examples of potentials $V_{n}$ with $\left|V_{n}\right|=O\left(n^{-1 / 2+\varepsilon}\right)$ and where $M$ has only point spectrum. Our discussion should be viewed as a remark on the recent work of Delyon, Kunz, and Souillard.
\end{abstract}

\section{Introduction}

During the past few years, numerous results have appeared showing that most of the spectrum of Schrödinger operators $-\Delta+V$ is purely absolutely continuous (a.c.). This includes not only the "short range case", $V(x)=O\left(x^{-1-\varepsilon}\right)$ at infinity (see e.g. [1]), but also the long range case where $V(x)=O\left(x^{-\varepsilon}\right)$ so long as derivatives decay (see e.g. [5]) and the highly oscillatory case where $V(x)=O\left(x^{-\alpha} \sin x^{\beta}\right)$ for suitable $\alpha, \beta$ (see e.g. [8]). While there are examples of Pearson [7] with decaying $V$ where the spectrum is not a.c., the rate is so slow that one might be led to suspect that reasonable (say power) decay always leads to a.c. spectrum. (The other recently constructed examples with non-a.c. behavior, namely random [4] and special almost periodic potentials [2], of course, have no decay at infinity.) Our goal here is to indicate that there are power decaying potentials which lead to nona.c. spectrum.

In the end our examples will be for $-\Delta$ replaced by a finite difference operator; explicitly, on $\ell^{2}(Z)$ let

$$
\left(M_{0} u\right)(i)=u(i+1)+u(i-1)
$$

and

$$
(V u)(i)=V(i) u(i) .
$$

We will consider $M_{0}+V$ on $\ell^{2}(Z)$. The potential $V$ will obey $V(n) \sim O\left(n^{-1 / 2+\varepsilon}\right)$, but differences $V(n+1)-V(n)$ will also be $O\left(n^{-1 / 2+\varepsilon}\right)$. The non-random analog will be potentials of the form $x^{-\alpha} \sin x$ with $\alpha<\frac{1}{2}$. It is an interesting fact that the known

* Research partially supported by USNSF under grant MCS 81-20833 
spectral properties of such Schrödinger operators is not pure point; rather it is mainly a.c. with some isolated eigenvalues [8].

Our main theorem is stated for almost all potentials in a random class of potentials. Our proofs will use the recent formalism of Delyon et al. [3], and this paper is essentially a remark on theirs.

Theorem 1.1. Let $\left\{W_{n}\right\}_{n=-\infty}^{\infty}$ be identically distributed independent random variables whose distribution is $r(x) d x$ with $r \in L^{1} \cap L^{\infty}$ with bounded support. Let $a_{n}$ be a fixed sequence with $0 \leqq a_{n} \leqq 1$ and $a_{n} \geqq C|n|^{-\alpha}$ for some $\alpha<\frac{1}{2}$. Let $V(n)=a_{n} w_{n}$. Then for a.e. w, $M_{0}+V$ has only point spectrum.

Remark. 1. $\sigma_{\text {ess }}\left(M_{0}+V\right)=[-2,2]$, so the point spectrum is dense in that interval. The eigenfunctions there will be shown to decay at least as fast as $O\left(\exp \left(-c|n|^{\beta}\right)\right)$, where $\beta=1-2 \alpha$, and this is probably the exact behavior if $a_{n} n^{\alpha} \rightarrow$ const.

2. The identity of the distribution of $w_{n}$ is not really important, but for the proof, independence is used heavily. In the end, the proof will go through as long as the distribution of $V(n)$ [note $V(n)$ not $w_{n}$; in our case, $V(n)$ has $\operatorname{dist} r_{n}(x) d x$, where $\left.r_{n}(x)=a_{n}^{-1} r_{n}\left(a_{n}^{-1} x\right)\right]$ is of the form $r_{n}(x) d x$, where (i) $\int r_{n}(x) d x=1$, (ii) $\left\|r_{n}\right\|_{\infty}=O\left(n^{m}\right)$ for some $m$, (iii) $\sup _{k \geq K_{0}}\left|\tilde{r}_{n}(k)\right| \leqq 1-n^{-2 \alpha}$ for some $D>0, \alpha<\frac{1}{2}$. Here $K_{0}$ is the constant of Theorem A.3 (say $K_{0}=0.3$ ) and $\tilde{r}_{n}(k)=\int e^{i k x} r(x) d x$. In particular, one can add a fixed potential to the above $V_{w}$ and still have only point spectrum.

3. If $\sum\left|a_{n}\right|<1$, then the trace class theory assures us that $M_{0}+V$ has lots of a.c. spectrum, so if $a_{n} \sim n^{-\alpha}$ with $\alpha>1$, then our result definitely does not hold. The behavior in the region $\frac{1}{2}<\alpha<1$ is open. Pearson's intuition [7] suggests that perhaps the key is $\sum\left(a_{n}\right)^{2}=\infty$ which suggests our $\alpha<\frac{1}{2}$ is the right condition.

Theorem 1.1 is proven in Sect. 2 by following the approach of [3] with minor changes. The necessary estimates are in [3], but we repeat them in an appendix, in part for the reader's convenience, and in part because the dependence of the estimates on $r_{n}$ is in one place only implicit in [3] and we need the explicit behavior.

\section{Proof of the Main Theorem}

In [6], Kunz and Souillard prove a criterion for a class of random operators on $\ell^{2}$ to have only point spectrum. The following is proven by their methods:

Theorem 2.1. Let $V_{w}(n)$ be a family of random potentials and let $M_{w}=M_{0}+V_{w}$. Define

$$
a(i, j)=E\left(\sup _{t}\left|\left(\delta_{i} e^{i t M(w)} \delta_{j}\right)\right|\right)
$$
where $\delta_{i}$ is the vector in $L^{2}$ which is 1 at $i$ and 0 elsewhere. If $\sum_{i}|a(i, j)|^{2}<\infty$ for
$i=0,1$, then for a.e. $w, M_{w}$ only has point spectrum.

Proof. As explained in [6], the RAGE theorem implies that $E\left(\left\|P_{\text {cont }} \delta_{j}\right\|^{2}\right)=0$ if $\sum_{i}|a(i, j)|^{2}<\infty$. Since polynomials in $M_{w}$ on $\delta_{0}, \delta_{1}$ are dense in $\ell^{2}$, we know that $P_{\text {cont }}=0$ if $\left\|P_{\text {cont }} \delta_{j}\right\|=0$ for $j=0,1$. 
For national convenience, we study $a(i, 0)$ with $i>0$. The discussion of $i \leqq 0$ and $a(i, 1)$ is essentially identical. In [3], Delyon et al. introduce the integral operators for $\alpha=0,1$ :

$$
\left[T_{\alpha}(p) f\right](x)=\int p\left(x+\frac{1}{y}\right) \frac{1}{|y|^{\alpha}} f(y) d y .
$$

For the case at hand, we will take $p(x)=r_{n}(e+x)=r_{n, e}(x)$, where $r_{n}(x)=a_{n}^{-1} r\left(a_{n}^{-1} x\right)$. We call these operators $T_{\alpha ; n, e}$. In $[3,6]$, the following result is proven using the operator $(\hat{U} f)(y)=\frac{1}{|y|} f\left(\frac{1}{y}\right)$ :

Theorem 2.2. Define for $N \geqq j$ :

$$
A(j, e ; N)=\left(\hat{U} T_{0 ; 0, e} T_{0:-1, e} \ldots T_{0 ;-N+1} r_{-N ; e}, T_{1 ; 1, e} \ldots T_{1 ; j-1, e} T_{0 ; j, e} \ldots T_{0 ; N-1, e} r_{N}\right)
$$

where $(\ldots, \ldots)$ is the $L^{2}(d x)$ inner product. Then

$$
a(j, 0) \leqq \lim _{N \rightarrow \infty} \int A(n, e ; N) d e,
$$

where the integral is over any set big enough to include the spectrum of all $M_{w}$.

In particular, if suppr $\subset[-B, B]$, we have

$$
a(j, 0) \leqq C \sup _{e, N \geqq j+1} A(j, e ; N) \equiv C A(j),
$$

with $C=4+2 B\left[\operatorname{since} \operatorname{spec}\left(M_{w}\right) C[-B-2, B+2]\right]$. If $\|\cdot\|_{p, q}$ is the norm of $\cdot$ as a map from $L^{p}$ to $L^{q}$, then since $r_{i}$ has $L^{1}$ norm 1 , since $\hat{U}$ is an isometry on $L^{2}$ and since $T_{0}$ is a contraction on $L^{2}$ [Theorem A.1(a)]:

$$
A(j) \leqq \sup _{e}\left[\left\|T_{0 ; 0, e}\right\|_{1,2}\left\|T_{1 ; 1, e} \ldots T_{1 ; j-1, e}\right\|_{2,2}\left\|T_{0 ; j, e}\right\|_{1,2}\right] .
$$

Define

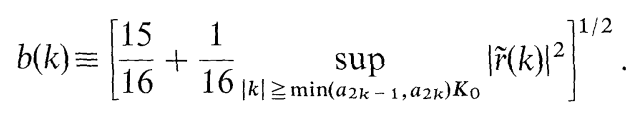

Using Theorems A.1(b) and A.3(a) and (c), we see that

$$
A(j) \leqq C a_{j}^{-1 / 2} b(1) \ldots b(\ell),
$$

if $j=2 \ell+1$ or $2 \ell+2$. Since $|\tilde{r}(k)|<1$ if $|k|=0$, and $|\tilde{r}(0)|=1$ with $\frac{d}{d k^{2}}|\tilde{r}(k)|<0$ [since it is essentially $\left.-\int x^{2} r(x) d x+\left(\int x r(x)\right)^{2}\right]$, we have for $\lambda$ small $\sup |r(k)|^{2} \leqq e^{-\gamma|\lambda|^{2}}$ for some $\gamma>0$, and thus

$$
|b(j)| \leqq \exp \left(-\tilde{\gamma} j^{-2 \alpha}\right)
$$

and

$$
|b(1) \ldots b(\ell)| \leqq \exp \left(-\gamma^{\prime} \ell^{1-2 \alpha}\right)
$$

This decay always wins out over the growth of $a_{j}^{-1 / 2}$ and yields enough decay to have $\sum A(j)^{2}<\infty$. Thus Theorems 2.1 and 2.2 yield Theorem 1.1. 


\section{Appendix: The Basic Estimates}

We define operator $T_{i}(r)$ on nice functions by:

and

$$
\left[\left(T_{0}(r)\right)(f)\right](x)=\int r\left(x+\frac{1}{y}\right) f(y) d y,
$$

$$
\left[\left(T_{1}(r)\right)(f)\right](x)=\int r\left(x+\frac{1}{y}\right) \frac{1}{|y|} f(y) d y .
$$

In this appendix we want to prove certain estimates which we emphasize are mainly in the paper of Delyon et al. [3]. We put them here in part for the reader's convenience, and in part because our proof of Theorem A.2 is an alternate to theirs which we prefer. Our main reason is to make the $r$ dependence of certain estimates explicit (since we need that) and to emphasize that the symmetry of $K U K$ which they use is not needed (and is not present in our case). We owe the argument in Theorem A.1(b) to A. Klein and C.Prakash.

Theorem A.1 [3].

(a) If $r \in L^{1}$, then $\left\|T_{0}(r) f\right\|_{1} \leqq\|r\|_{1}\|f\|_{1}$.

(b) if $r \in L^{1} \cap L^{\infty}$, then $\left\|T_{0}(r) f\right\|_{2} \leqq\|r\|_{\infty}^{1 / 2}\|r\|_{1}^{1 / 2}\|f\|_{1}$.

Proof. (a) is trivial if we first integrate $d x$ and then $d y$; indeed for $r, f \geqq 0$, we have equality.

$$
\begin{aligned}
\left\|T_{0}(r) f\right\|_{2}^{2} & \leqq \int\left|r\left(x+\frac{1}{y}\right)\right|\left|r\left(x+\frac{1}{z}\right)\right||f(y)||f(z)| d y d z d x \\
& \leqq\|r\|_{\infty} \int\left|r\left(x+\frac{1}{y}\right)\right||f(y)||f(z)| d y d z d x \\
& =\|r\|_{\infty}\|r\|_{1}\|f\|_{1}^{2} \cdot \square
\end{aligned}
$$

To analyze $T_{1}$, we follow Delyon et al. [3] (modulo a sign) and write

$$
T_{1}(r)=K(r) U \text {, }
$$

where

and

$$
(U f)(x)=\frac{1}{|x|} f(-1 / x)
$$

$$
(K(r) f)(x)=\int r(x-y) f(y) d y .
$$

Let

$$
\tilde{r}(k)=\int r(x) e^{-i k x} d x,
$$

and let $\mathscr{F}$ be the conventional Fourier transform. Define $\tilde{K}(r)=\mathscr{F} K(r) \mathscr{F}^{-1}$, $\tilde{U}=\mathscr{F} \underset{\tilde{K}}{\mathscr{F}^{-1}}$. Since $\mathscr{F}$ is unitary on $L^{2}$, to study $L^{2}$ properties of $T_{1}$, we need only study $\tilde{K} \tilde{U}$. Obviously

To study $\tilde{U}$ we note

$$
(\tilde{K}(r) g)(k)=\tilde{r}(k) g(k) \text {. }
$$

Lemma A.2. $\tilde{U}$ has an integral kernel (say in distributional sense) $a(k, p)$, where

(a) $a=a_{1}+a_{2}$ with $\sup _{k} \int\left|a_{1}(k, p)\right|^{2} d p<\infty$ and $\sup _{p} \int\left|a_{2}(k, p)\right|^{2} d k<\infty$.

(b) For any $R, \int_{|k| \leqq R,|p| \leqq R}|a(k, p)|^{2} d p d k<\infty$. 
Proof. (b) follows from (a). For proving (a), we note that

$$
a(k, p)=(2 \pi)^{-1} \int e^{i\left(k x+p x^{-1}\right)} \frac{d x}{|x|}
$$

(in distributional sense). Let $g_{1}$ be in $C_{0}^{\infty}$ with $g_{1} \equiv 1$ near 0 , and let $g_{2}=1-g_{1}$. Define

$$
a_{i}(k, p)=(2 \pi)^{-1} \int e^{i\left(k x+p x^{-1}\right)} g_{i}(x)|x|^{1} d x .
$$

For fixed $p, a_{2}(k, p)$ is the Fourier transform of $(2 \pi)^{-1 / 2} e^{i p x^{-1}} g_{2}(x)|x|^{-1}$, which is in $L^{2}$, so

$$
\int\left|a_{2}(k, p)\right|^{2} d p=(2 \pi)^{-1} \int\left|g_{2}(x)\right|^{2} x^{-1} d x<\infty
$$

independently of $p$. By changing variables from $x$ to $x^{-1}$, we see that

$$
a_{1}(k, p) \equiv(2 \pi)^{-1} \int e^{i\left(k y^{-1}+p y\right)} g_{1}\left(y^{-1}\right)|y|^{-1} d y,
$$

so

$$
\int\left|a_{1}(k, p)\right|^{2} d k=(2 \pi)^{-1} \int\left|g_{1}\left(y^{-1}\right)\right|^{2} y^{-2} d y<\infty
$$

independently of $p$.

Theorem A.3 [3].

(a) $\left\|T_{1}(r) f\right\|_{2} \leqq\|f\|_{2}$, if $\|r\|_{1}=1$.

(b) If $r, q \in L^{1} \cap L^{2}$, then $T_{1}(r) T_{1}(q)$ is compact.

(c) For some fixed $K_{0}$ and $r, q$ with $\|r\|_{1}=\|q\|_{1}=1$; $\|r\|_{2}<\infty,\|p\|_{2}<\infty$

$$
\left\|T_{1}(r) T_{1}(q) f\right\|_{2} \leqq A\|f\|_{2},
$$

where

$$
A=\operatorname{ax}\left[\left(\frac{15}{16}+\frac{1}{16} \sup _{|k| \geqq K_{0}}|\tilde{r}(k)|^{2}\right)^{1 / 2},\left(\frac{15}{16}+\frac{1}{16} \sup _{|k| \geqq K_{0}}|\tilde{q}(k)|^{2}\right)^{1 / 2}\right] .
$$

Proof. (a) is trivial since $U$ is unitary and $K$ is a contraction if $\|r\|_{1}=1$.

(b) Since $U$ is unitary, we need only show that $\tilde{K}(r) \tilde{U} \tilde{K}(q)$ is compact. Since $\tilde{r} \in L^{2}$ and $\tilde{q} \in L^{\infty}, \quad \tilde{r}(k) a_{1}(k, p) \tilde{q}(p) \in L^{2}\left(R^{2}\right)$ and similarly, since $\tilde{r} \varepsilon L^{\infty}, \tilde{q} \varepsilon L^{2}$, $\tilde{r}(k) a_{2}(k, p) \tilde{q}(p) \in L^{2}\left(R^{2}\right)$, so $\tilde{K}(r) U \tilde{K}(q)$ is Hilbert-Schmidt.

(c) Since $U$ is unitary, we need only show that if $\|\varphi\|_{2}=\|\psi\|_{2}=1$, then

$$
|(\varphi, \tilde{K}(r) U \tilde{K}(q) \psi)| \leqq A
$$

Pick $K_{0}$ so that

$$
\left.\int_{\substack{|k| \leqq K_{0} \\|q| \leqq K_{0}}}|a(k, q)|^{2} d k d q\right)^{1 / 2} \leqq \frac{7}{16},
$$

which is possible since the left side goes to zero as $K_{0} \downarrow 0$ (indeed, if one takes $g_{1}$ to be the characteristic function of $[-1,1]$, one sees that one can choose

$$
\begin{aligned}
& K_{0}=49 \pi / 512 \simeq 0.3 \text { ). If } \int_{|k| \geqq K_{0}}|\psi(k)|^{2} d k \geqq \frac{1}{16} \text {, then } \\
& \|\tilde{K}(g) \psi\|^{2} \leqq\left(\frac{15}{16}+\frac{1}{16} \sup _{|k| \geqq K_{0}}|\tilde{q}(k)|^{2}\right) \leqq A^{2},
\end{aligned}
$$


so (A.7) certainly holds since $U$ and $\tilde{K}(r)$ have norm 1 . Similarly, (A.7) holds if $\int_{|k| \geqq K_{0}}|\varphi(k)|^{2} d k \geqq \frac{1}{16}$.

Thus, we only need to check (A.7) if $\left\|\varphi_{+}\right\| \leqq \frac{1}{4},\left\|\psi_{+}\right\| \leqq \frac{1}{4}$, where $\varphi_{+}=\varphi \chi_{\left\{|k| \geqq K_{0}\right\}^{\circ}}$ Let $\varphi_{-}=\varphi-\varphi_{+}$and note that by (A.8) and the fact that $\tilde{K}(\cdot)$ preserves supports and pointwise decreases values (if $\|\cdot\|_{1}=1$ ),

$$
\left(\varphi_{-}, \tilde{K}(r) U \tilde{K} \psi_{-}\right) \leqq 7 / 16,
$$

where we have used the fact that the norm of an operator is dominated by its Hilbert-Schmidt norm. Thus

$$
\begin{aligned}
|(\varphi, \tilde{K} U \tilde{K} \psi)| & \leqq\left\|\varphi_{+}\right\|+\left|\left(\varphi_{-}, \tilde{K} U \tilde{K} \psi\right)\right| \\
& \leqq\left\|\varphi_{+}\right\|+\left\|\psi_{+}\right\|+\left|\left(\varphi_{-}, \tilde{K} U \tilde{K} \psi_{-}\right)\right| \\
& \leqq \frac{1}{4}+\frac{1}{4}+\frac{7}{16}=\frac{15}{16}<A^{2}<A
\end{aligned}
$$

as required.

Remarks. 1. (b) is not used to estimate (c). We include it since it can be used to provide a computationless proof that $\left\|T_{1}(r) T_{1}(q)\right\|<1$ (see [3]).

2. If $r, q$ are fixed positive with $L^{1}$ norm 1 , and $r_{\lambda}(x)=r\left(\lambda^{-1} x\right) \lambda^{-1}$ so $\tilde{r}_{\lambda}(k)$ $=\tilde{r}(\lambda k)$, then (A.5) says that $\left\|T_{1}\left(r_{\lambda}\right) T_{1}\left(q_{\lambda}\right)\right\| \leqq 1-O\left(\lambda^{2}\right)$ as $\lambda \downarrow 0$. We claim that also $\left\|T_{1}\left(r_{\lambda}\right) T_{1}\left(q_{\lambda}\right)\right\| \geqq 1-O\left(\lambda^{2}\right)$ so that $O\left(\lambda^{2}\right)$ is precisely the correct behavior. For translating, we can suppose without loss that $\int x r(x) d x=\int x q(x) d x=0$. Then

$$
K\left(r_{\lambda}\right) g=g+O\left(\lambda^{2}\right)
$$

if $g \in C_{0}^{\infty}$. Choosing $g$ in $C_{0}^{\infty}$ with $\bigcup g=g$, we find that $T_{1}\left(r_{\lambda}\right) T_{1}\left(q_{\lambda}\right) g=g+O\left(\lambda^{2}\right)$, so $\left\|T_{1}\left(r_{\lambda}\right) T_{1}\left(q_{\lambda}\right)\right\| \geqq 1-O\left(\lambda^{2}\right)$.

Acknowledgements. It is a pleasure to thank B. Souillard for explaining [3] to me, and for encouragement, and to thank the members of the Caltech-Irvine random working seminar for valuable discussions.

\section{References}

1. Agmon, S.: Ann. Scu. Norm. Sup. Pisa 2, 151-218 (1975)

2. Avron, J., Simon, B.: Bull. Am. Math. Soc. 6, 81-85 (1982)

3. Delyon, F., Kunz, H., Souillard, B.: Ecole Polytechnique Preprint

4. Goldsheid, I., Molchanov, S., Pastur, L. : Funkt. Anal. Pril. 11, 1 (1977)

5. Kitada, H.: J. Math. Soc. Jpn. 29, 665-691 (1977); 30, 603-632 (1979)

6. Kunz, H., Souillard, B. : Commun. Math. Phys. 78, 201 (1980)

7. Pearson, D.: Commun. Math. Phys. 60, 13-36 (1978)

8. White, D.: Trans. Am. Math. Soc. (to appear)

Communicated by T. Spencer

Received June 28, 1982 